\title{
CULTURA ORGANIZACIONAL E ADOECIMENTO NO TRABALHO: UMA REVISÃO SOBRE AS RELAÇÕES ENTRE CULTURA, BURNOUT E ESTRESSE OCUPACIONAL
}

\author{
ORGANIZATIONAL CULTURE AND ILLNESS AT WORK: A REVIEW ON THE \\ RELATIONS AMONG CULTURE, BURNOUT AND OCCUPATIONAL STRESS
}

Carolina Villa Nova Aguiar', Eliana Edington da Costa Silva ${ }^{2}$, Bárbara Ribeiro de Carvalho ${ }^{3}$,
Jaqueline Cristina Muricy Ferreira ${ }^{3}$, Kaike Costa Oliveira de Jesus $^{4}$ 'Doutora em Psicologia. Professora na Escola Bahiana de Medicina e Saúde Pública. Salvador, Bahia, Brasil. ${ }^{2}$ Doutora em Psicologia Organizacional. Professora na Escola Bahiana de Medicina e Saúde Pública. Salvador, Bahia, Brasil. ${ }^{3}$ Acadêmica de Psicologia na Escola Bahiana de Medicina e Saúde Pública. Salvador, Bahia, Brasil.

${ }^{4}$ Acadêmico de Psicologia na Escola Bahiana de Medicina e Saúde Pública. Salvador, Bahia, Brasil.

RESUMO I O presente trabalho propõe-se a realizar uma revisão narrativa da literatura nacional dos últimos 15 anos (entre os anos de 2000 e 2015) sobre as relações entre a cultura organizacional e dois conceitos intimamente relacionados ao adoecimento do trabalhador: o estresse ocupacional e o burnout. Para isso, foi realizado um levantamento bibliográfico por meio da consulta às bases de dados LILACS, PePSIC e SciElo. De forma complementar, foi consultado o banco de teses da CAPES. No total, foram contemplados oito trabalhos científicos, sendo cinco referentes às relações entre cultura e estresse ocupacional e três sobre as relações entre cultura e burnout. Os resultados apontam a escassez de estudos brasileiros sobre o tema. Ademais, um aspecto importante refere-se à dificuldade de articulação dos estudos, o que pode ser atribuído, em alguma medida, à utilização de diferentes ferramentas para a apreensão dos mesmos fenômenos. Apesar dos desafios gerados pelas inconsistências empíricas, foi possível identificar que a cultura organizacional configura-se como um preditor do desenvolvimento do estresse ocupacional e da síndrome de burnout. Acredita-se que $\circ$ presente trabalho poderá contribuir para a elaboração de novas políticas e práticas organizacionais que prezem pela qualidade da cultura desenvolvida dentro do âmbito laboral.

Palavras-chave: cultura organizacional; estresse ocupacional; síndrome de burnout.

\begin{abstract}
I This paper proposes to carry out a narrative review of the national literature of the last 15 years (between 2000 and 2015) on the relationship between organizational culture and two closely related concepts with the employee's illness: occupational stress and burnout. For this, we conducted a literature survey by consulting the databases LILACS, PePSIC, SciElo and the bank of theses (CAPES). In total, eight scientific works were awarded: five about the relations between culture and occupational stress and three about the relationship between culture and burnout. The results indicate the lack of Brazilian studies on the subject. In addition, an important aspect is the difficulty of joint studies, which can be attributed, in some measures, to the use of different instruments for the measure of the same phenomena. Despite the challenges generated by empirical inconsistencies, it was identified that the organizational culture can predict occupational stress development and burnout syndrome. It is believed that this study may contribute to the development of new policies and organizational practices that invest in the quality of organizational culture.
\end{abstract}

Key-words: organizational culture; occupational stress; burnout. 


\section{INTRODUÇÃO}

O trabalho se configura como um importante eixo organizador da vida de muitos indivíduos. Como consequência, percepções e acontecimentos oriundos do contexto organizacional apresentam grande potencial para impactar diretamente em diversas variáveis que ocorrem no nível individual, como, por exemplo, aquelas relacionadas ao adoecimento mental do trabalhador. Corroborando com essa lógica, diversos estudos têm se dedicado à compreensão sobre o papel desempenhado por diferentes aspectos do ambiente de trabalho na promoção de saúde ou de sofrimento psíquico dos indivíduos que nele se encontram (Codo, Soratto, \& Vasques-Menezes, 2004; Maslach, 2007; Tamayo, Lima, \& Silva, 2004). Entre as variáveis contextuais presentes em todas as organizações, encontra-se a cultura organizacional, que tem sido consensualmente tratada como um fenômeno com elevado grau de complexidade e multifacetado, composto por diferentes elementos que, em conjunto, apresentam grande potencial para influenciar no dia a dia dos trabalhadores dentro de uma organização (Cunha, 2014; Schein, 2009; Silva, 2015; Silva, Zanelli, \& Tolfo, 2013).

Diante do exposto, considera-se que a cultura organizacional representa um fenômeno que pode contribuir ativamente para a promoção de saúde mental - quando percebida como acolhedora e saudável-ou para o desencadeamento de sofrimento psíquico - quando avaliada como pouco acolhedora ou até mesmo patológica - dos trabalhadores. O presente trabalho, portanto, propõe-se a realizar uma revisão da literatura nacional sobre as relações entre a cultura organizacional e dois conceitos intimamente relacionados ao adoecimento do trabalhador: 0 estresse ocupacional e o burnout. Acredita-se que oferecer uma maior sistematização do conhecimento até então produzido sobre essas relações poderá subsidiar a construção de políticas e práticas organizacionais que prezem pela qualidade da cultura desenvolvida dentro do âmbito laboral.

\section{CULTURA ORGANIZACIONAL}

A temática denominada cultura organizacional traz consigo especificações que englobam raízes na antropologia, referindo-se a explicações de fatos cotidianos. Inicialmente, as investigações sobre o tema estavam totalmente desconectadas do mundo empresarial, sendo realizadas exclusivamente em sociedades tribais. Apenas a partir da década de 1980, a cultura das organizações começa a atrair os olhares de acadêmicos e profissionais, em especial aqueles inseridos na área da educação (Cunha, 2014).

Durante muitos anos, diversos pesquisadores empreenderam esforços na busca de uma conceituação uniforme sobre cultura organizacional (Cunha, 2014; Schein, 2009). No entanto, as tentativas foram frustradas pelo fato de existir uma vasta nomenclatura nesta área de conhecimento, - que corroborou ainda mais para uma confusão conceitual sobre este tema. Sobre essa dificuldade de se alcançar uma conceituação clara e consensual para definir o que é cultura organizacional, Schein $(2009$, p. 11$)$ afirma que:

(...) Temos uma noção desse fenômeno oriunda do senso comum, mas é difícil defini-lo abstratamente. Ao conversar sobre cultura organizacional com colegas e membros de organizações, frequentemente constato que concordamos que "ela" existe e que é importante em seus efeitos, mas quando tentamos defini-la, temos

ideias completamente diferentes do que "ela" é.

Ainda que reconheça a dificuldade de se definir precisamente $\bigcirc$ que é cultura organizacional, Schein (2009) propõe que ela seja formalmente conceituada como um conjunto de suposições básicas compartilhadas que estabelecem um padrão, considerado correto, de perceber, pensar e sentir-se em relação a diferentes aspectos da organização Nota-se, portanto, que a cultura representa um fenômeno dinâmico, trazendo consigo um conjunto de normas, regras, estruturas, e rotinas que orientam os comportamentos dos indivíduos no trabalho.

No entanto, para que a compreensão acerca da cultura organizacional seja completa, torna- 
se imprescindível se levar em consideração que se trata de um fenômeno multifacetado, sendo composto por três elementos distintos que diferem em termos de facilidade de apreensão e, também, em profundidade: os artefatos visíveis, os valores e os pressupostos básicos (Motta, 1995; Schein, 2009; Silva, 2015; Silva et al., 2013). Os artefatos visíveis, situados no primeiro nível, correspondem a processos e outros fenômenos organizacionais facilmente observáveis (visíveis) como por exemplo, - layout da instituição, as vestimentas, a linguagem da organização, sendo desta forma, de difícil interpretação. No segundo nível, encontram-se os valores, que representam as crenças, muitas vezes presentes nos discursos oficiais dos representantes da organização, na comunicação institucional, racionalizações ou aspirações para o futuro. Por fim, os pressupostos ou premissas básicas, situados no terceiro e mais profundo nível, representam as concepções que estão tão internalizadas pelos atores organizacionais que direcionam as suas atitudes e seus comportamentos de forma imperceptível. São os pressupostos básicos que explicam o comportamento real (não o esperado) dos membros de uma organização (Silva et al., 2013).

Além dos diversos níveis, há, ainda, diferentes modelos para apreensão da cultura. Entre eles, o mais popular e hegemônico é o proposto por Geert Hofstede, que considera que a cultura organizacional não deve ser compreendida à parte da cultura nacional na qual está inserida (Cunha, 2014; Zanelli, \& Silva, 2014). A partir dessa constatação, o pesquisador desenvolveu um quadro-referência que descreve diferentes dimensões da cultural nacional que podem ser transpostas para a classificação da cultura das organizações (Figura 1).

Quadro 1. Modelo teórico de Hofstede de cultura organizacional

\begin{tabular}{|c|c|}
\hline Dimensão & Definição \\
\hline $\begin{array}{l}\text { Individualismo } \\
\text { vs } \\
\text { Coletivismo }\end{array}$ & $\begin{array}{l}\text { Indica se uma sociedade é uma rede social sem relação entre os indivíduos, } \\
\text { em que pessoas tomam conta apenas de si próprias e daqueles que lhes } \\
\text { estão mais próximas, ou em contrapartida se é caracterizada por redes } \\
\text { sociais profundas, nas quais as pessoas fazem distinção entre seus próprios } \\
\text { grupos, esperando seus grupos deem proteção aos seus membros. }\end{array}$ \\
\hline Distância do poder & $\begin{array}{l}\text { Refere-se ao quanto os membros menos poderosos da organização aceitam } \\
\text { a distribuição desigual de poder. Em um país que a distância de poder é } \\
\text { alta, tanto subordinados como superiores pensam que passar por cima de um } \\
\text { nível hierárquico é uma insubordinação. Em contrapartida, países com baixa } \\
\text { distância de poder, tem tendência dos empregados saltarem niveis } \\
\text { hierárquicos com intuito de realizar melhor seu trabalho. }\end{array}$ \\
\hline Evitação da incerteza & $\begin{array}{l}\text { Caracteriza o grau de ameaça percebido por membros de uma cultura } \\
\text { diante de situações ambíguas, bem como a forma pela qual essas situações } \\
\text { são evitadas, críando assim maior estabilidade de carreira. }\end{array}$ \\
\hline $\begin{array}{l}\text { Masculinidade } \\
\text { vs } \\
\text { Feminilidade }\end{array}$ & $\begin{array}{l}\text { Refere-se até que ponto a cultura enfatiza tanto a assertividade quanto a } \\
\text { aquisição de dinheiro e das coisas versus uma cultura que salientam os } \\
\text { relacionamentos entre pessoas, a preocupação com o próximo e a qualidade } \\
\text { de vida global. }\end{array}$ \\
\hline Pragmatismo & $\begin{array}{l}\text { Diz respeito ao grau em que as pessoas consideram que ocorre em seu redor } \\
\text { não pode ser explicado. E a dimensão indulgência reflete o grau em que as } \\
\text { pessoas tentam controlar seus desejos e impulsos }\end{array}$ \\
\hline
\end{tabular}

FONTES: Cunha $^{4}$ e Motta ${ }^{8}$.

Embora não esteja isento de críticas, o modelo de G. Hosfstede tem sido amplamente adotado como referência para o estudo sobre a cultura organizacional (Zanelli, \& Silva, 2014). De acordo com Cunha (2014), as suas implicações para a área de gestão são nítidas, uma vez que evidenciam a necessidade de as políticas e práticas organizacionais estarem congruentes com o contexto ambiental maior, ou seja, a cultura nacional.

\section{ESTRESSE NO TRABALHO}

A concepção de estresse como fenômeno psicossocial ganhou visibilidade na década de 1930, a partir dos resultados dos trabalhos produzidos por Hanz Selye, passando a ser reconhecido pela medicina e biologia, e, posteriormente, também pela psicologia (Helman, 1994; Gasparini, \& Rodrigues, 1992). Após a Segunda Guerra Mundial, houve um considerável 
crescimento do interesse sobre o estresse por parte de psicólogos e psiquiatras, que desenvolveram trabalhos científicos sobre os impactos da guerra nos indivíduos. Nesta época, os estudos de autores como Kahn, Wolfe, Quinn, Snoek e Rosenthal foram os que mais ganharam visibilidade, tendo em vista que estudaram o estresse a partir de fatores como ambiguidade, conflito de papéis e sobrecarga (Canova, \& Porto, 2010).

Etimologicamente, estresse deriva do latim stringere, que significa apertar, cerrar, comprimir (Hovaiss, Villar, \& Franco, 2001). Lippi (2001 apud Tavares, Tadeucci, \& Inocente, 2011, p.3) define 0 estresse como:

Uma reação do organismo com componentes físicos e/ou psicológicos, causada pelas alterações psicofisiológicas que ocorrem quando a pessoa se confronta com uma situação que, de um modo ou de outro, a irrite, amedronte, excite ou confunda, ou mesmo que a faça imensamente feliz.

A partir da definição oferecida, fica nítido o caráter não específico da definição do estresse, o que, em parte, explica o seu uso nos mais diversos contextos, contribuindo assim, para a sua grande propagação. Nota-se, portanto, que a denominação estresse converteu-se em uma palavra comum, que popularmente representa qualquer tipo de angústia ou cansaço do corpo e da mente. Independente do uso, as pessoas referem-se ao termo quando querem dizer que estão aflitas, cansadas ou irritadas. Entretanto, mais do que um estado de espírito, o estresse deve ser compreendido como uma reação do indivíduo a uma situação que exija adaptação que, quando realizada de modo insatisfatório, pode causar um conjunto de sintomas, sejam eles físicos, psicológicos e/ou comportamentais (Zanelli, \& Silva, 2014).

Diferentemente do que se pensa no senso comum, Nelson e Simmons (2012) mencionam que o estresse tem um papel importante e funcional, sendo necessário para movimentar as pessoas, dando forças para alcançarem seus objetivos. Contudo, quando vivenciado em excesso, elimina as defesas do corpo, afetando a saúde do indivíduo. Assim, o estresse não é essencialmente ruim, embora, em geral, seja inserido em um contexto negativo.
Com o crescimento do interesse sobre o fenômeno do estresse, Lipp (2001 apud Tavares et al., 2011) considerou ser fundamental registrar as diferenças entre os aspectos relacionados ao estresse ocasionado pelas situações da vida cotidiana e o estresse oriundo de contextos específicos, como é o caso do estresse ocupacional. $O$ estresse ocupacional, definido como uma reação mediante as demandas sociopsicológicas, caracteriza-se como um possível indicador das consequências do trabalho sobre os indivíduos, já que os mesmos podem estar em estado de sofrimento devido às condições e características de sua atividade profissional, sem necessariamente apresentar nenhum quadro patológico definido (Tavares et al., 2011).

De acordo com Mussolin e Rodas (2013, p.93), pode-se definir estresse no trabalho como:

As reações físicas e emocionais que ocorrem quando as exigências do trabalho não igualam as suas capacidades, ou as necessidades do trabalhador. $\bigcirc$ estresse no trabalho pode ocorrer da relação entre o indivíduo e o seu ambiente de trabalho no qual as exigências do trabalho ultrapassam as habilidades do trabalhador para enfrentá-las, podendo acarretar um desgaste excessivo do organismo, interferindo na sua produtividade.

De acordo com Tavares et al. (2011), o papel do estresse organizacional na sociedade tornou-se uma fonte importante de preocupação. Isso se faz notório pelo fato desta especificidade possuir estreita relação com uma série de doenças que acometem grande quantidade de trabalhadores, como por exemplo: obesidade, doenças osteomusculares, cardiovasculares, diabetes, além de distúrbios sociopsíquicos como ansiedade e depressão. Além disso, pessoas que sofrem com estresse no trabalho normalmente são improdutivas e tornam-se facilmente irritáveis e até mesmo deprimidas.

No ambiente laboral, aspectos como elevada carga horária de trabalho e relações hierárquicas, podem se configurar como potenciais agentes estressores. Assim, corroborando com esta afirmativa, Spector (2002, p. 292) considera que "os modelos de processos de stress no trabalho presumem que os fatores estressantes levam ao desgaste. No entanto, sabe-se que o processo não é automático, e que as percepções e a avaliação dos funcionários sobre 
esse fator são parte essencial do processo".

De acordo com os estudos de Santos e Cardoso (2010) sobre o estresse em profissionais de saúde, o evento estressor em si não oferece subsídios suficientes para abarcar toda a reação de estresse. Essa afirmativa corrobora o estudo de Mussolin e Rodas (2013), que aponta ser extremamente importantes a avaliação e a interpretação do indivíduo frente aos eventos estressantes, tendo em vista que este utiliza os mais variados recursos internos e externos como formas estratégicas direcionadas ao enfrentamento das demandas desencadeadoras de estresse. Tamayo et al. (2004) também enfatizam a importância de se considerar as relações estabelecidas entre o trabalhador, o evento estressante, 0 ambiente de trabalho e, ainda, a maneira como o indivíduo encara a situação. Ou seja: os autores destacam que o estresse não é um fator exclusivo do trabalhador, nem do ambiente de trabalho, sendo, ao contrário, um fator que emerge das relações do empregado com seu ambiente de trabalho.

A partir de estudos com trabalhadores brasileiros, Soares (2010) identificou três fontes principais de estresse no trabalho: 1) relação com empresa: refere-se a percepções de responsabilidades excessivas, dificuldades na relação com os chefes, falta de estímulos, promoções e planejamento por parte dos gestores; 2) relação com os colegas: relaciona-se com a avaliação de que os colegas não cooperam $\circ$ suficiente para $\circ$ bom funcionamento das atividades, além de questões ligadas a falta de união entre os funcionários; 3 ) relação com o próprio modo de ser: diz respeito a características disposicionais do funcionário, como o grau de auto cobrança, as expectativa geradas etc.

\section{BURNOUT}

O conceito de Burnout surge pela primeira vez na literatura na década de 1970, introduzido pelo psiquiatra e psicanalista norte-americano Herbert Freudenberg, que, ao estudar muitos profissionais voluntários, constatou que estes apresentavam um desgaste do humor e de motivação. A partir desses estudos, o Burnout passou a ser compreendido como um conjunto de sintomas, tanto biológicos quanto psicossociais, que são consequência de uma exigência excessiva de energia a ser direcionada ao trabalho (Guimarães, \& Cardoso, 2004).

Outros autores prosseguiram com estudos sobre a temática e, hoje, é possível identificar quatro perspectivas distintas para a compreensão desse mesmo fenômeno. Uma primeira perspectiva é a clínica, desenvolvida pelo próprio $\mathrm{H}$. Freudenberg, que considera sintomas biológicos, físicos e psíquicos para caracterizar o burnout, sendo que tais sintomas podem acarretar em uma depressão ou em suicídio. É possível notar, aqui, que a perspectiva clínica foca unicamente nas características individuais, representando uma visão unidimensional da síndrome, desconsiderando a dimensão social (Pereira, 2002; Tironi, 2005).

Uma segunda perspectiva é a organizacional, que compreendeoBurnoutcomoresultadoda inadequação entre as vontades do trabalhador e os interesses da organização (Pereira, 2002; Tironi, 2005). Nesse caso, agentes estressores organizacionais seriam responsáveis pelo desencadeamento de mecanismos de enfrentamento por parte dos trabalhadores. Contudo, tais mecanismos, a partir de determinado momento, passam a ser disfuncionais e favorecerem o surgimento do Burnout (Pereira, 2002).

A terceira perspectiva, denominada sócio-histórica, enfatiza $\circ$ determinante papel da sociedade moderna, caracterizada pelo individualismo e competição, no desenvolvimento da síndrome. De tal modo, ocupações voltadas para a ajuda e $\circ$ desenvolvimento do próximo (perspectiva comunitária), são incompatíveis com os valores predominantes na sociedade atual, a qual não prioriza os fatores pessoais ou institucionais (Pereira, 2002; Tironi, 2005).

Por fim, a perspectiva sócio-psicológica, que tem sido amplamente adotada em pesquisas recentes, enfatiza os fatores socioambientais no processo de desenvolvimento do Burnout, destacando, assim, o caráter multideterminado da síndrome, que passa a ser compreendida como uma reação frente à exposição prolongada a estressores interpessoais crônicos (Maslach, 2007; Mallmann, Palazzo, Carlotto, \& Castro, 2009; Pereira, 2002; Tironi, 2005). De acordo com o modelo proposto por Cristina Maslach e Suzan Jackson, principais 
desenvolvedoras dessa perspectiva, a síndrome de Burnout se manifesta através de três dimensões distintas, sendo elas:

a) Exaustão Emocional: refere-se ao sentimento de estar emocionalmente sobrecarregado e desprovido de recursos emocionais (Marques, 2011 ). Caracteriza-se por sensações de estar além dos limites, não dispondo de energia para realizar nada, bem como esgotamento físico e emocional, sem qualquer fonte de reposição (Maslach, 2007; Pereira, 2002; Tironi, 2005). As principais fontes desse esgotamento são a sobrecarga e o conflito pessoal no trabalho associado ao sentimento de não serem recompensados (Marques, 2011; Maslach, 2007).

b) Despersonalização: é o componente do contexto interpessoal do Burnout, no qual $\circ$ profissional passa a ter reações negativas, insensiveis ou excessivamente desligadas, passando a denotar atitudes de cinismo e ironia em relação às pessoas $e$ indiferença ao que pode vir a acontecer aos demais (Marques, 2011; Maslach, 2007; Pereira, 2002; Tironi, 2005). Em geral, desenvolve-se, inicialmente, em resposta à sobrecarga de exaustão emocional (Maslach, 2007), que inicialmente surge como forma de autoproteção ou escape emocional que está ligado ao desprendimento, contudo há o risco de se tornar em desumanização (Marques, 2011 ).

c) Reduzida Realização Profissional: refere-se ao componente de auto-avaliação no Burnout, que evidencia o declínio do sentimento de competência e produtividade no trabalho (Marques, 2011; Maslach, 2007), bem como insatisfação com atividades laborais, baixa autoestima, sentimento de insuficiência, desmotivação, podendo culminar no desejo de abandonar o trabalho (Pereira, 2002; Tironi, 2005). Essas sensações são decorrência de falhas de suporte social e de oportunidades de desenvolvimento profissional, o que pode resultar em sentimentos de fracasso por parte do trabalhador em relação a si próprio (Marques, 2011 ; Maslach, 2007).

O Burnout, em geral, é mais facilmente observado em ocupações relacionadas a cuidados pessoais e serviços assistenciais como atendimento à saúde, saúde mental, assistência social, sistema judiciário penal, profissões religiosas, aconselhamento e ensino. Essas profissões têm em comum o fornecimento de auxílio e prestação de serviços a pessoas necessitadas, assim era estudado em termos de transações relacionais de um indivíduo no local de trabalho (Maslach, 2007). De acordo com Loureiro, Oliveira e Pessoa (2008, p. 34), "quando o local de trabalho é perspectivado como hostil, excessivamente exigente tanto econômica quanto psiquicamente, desenvolve-se uma crescente exaustão emocional, psíquica e espiritual". Além dos impactos negativos para o próprio indivíduo, a síndrome acarreta em consequências que podem ser percebidas nos níveis social e organizacional, já que, além do desgaste emocional, a síndrome conduz a um esgotamento dos relacionamentos sociais e familiares e, ainda, à redução do desempenho organizacional, o que se traduz em elevados custos para organização (Marques, 2011).

No que diz respeito à prevalência da síndrome na população, o que se nota é um crescimento contínuo do Burnout (Loureiro et al., 2008). Os autores afirmam que o número crescente de trabalhadores com a síndrome não se dá por comportamentos inadequados dos trabalhadores, mas sim da própria natureza do trabalho e das alterações ocorridas nos ambientes e relações laborais. Características estruturais da organização também podem favorecer o surgimento da síndrome. Lousada (2001 apud Loureiro et al., 2008) aponta, por exemplo que, quando o trabalho é muito formal, há tendência a se tornar rotineiro e monótono, enquanto que a informalidade pode gerar conflitos de papeis, sendo os dois potenciais propiciadoras ao aparecimento do Burnout.

\section{MÉTODO}

A presente pesquisa teve um caráter de revisão narrativa da literatura nacional dos últimos 15 anos (período de 2000 a 2015). Para o levantamento bibliográfico sobre tema pesquisado, foram consultadas as bases de dados LILACS, PePSIC e SciElo, usando os descritores "cultura", "cultura organizacional", "estresse", "estresse ocupacional" e "burnout" de formas independentes e combinadas. 
De forma complementar, foi consultado o banco de teses da CAPES, com o objetivo de identificar dissertações de mestrado e/ou teses de doutorado sobre o tema de interesse. Foram selecionados aqueles trabalhos que apresentaram os descritores no títulos ou no resumo. Todas as buscas foram realizadas no período de março a novembro de 2015.

\section{RESULTADOS E DISCUSSÃO}

Nesta seção, serão apresentados os resultados referentes à revisão de literatura. Como pode ser observado no Figura 2 um total de oito artigos cumpriram os critérios de inclusão e, portanto, foram contemplados na revisão de literatura nacional da área. Dos oito artigos, cinco versam sobre as relações estabelecidas entre a cultura organizacional e - estresse ocupacional e apenas três dedicamse à investigação sobre a cultura e suas possíveis associações com a síndrome do Burnout.

Quadro 2. Síntese dos estudos que compuseram a revisão

\begin{tabular}{|c|c|c|c|c|c|}
\hline \multicolumn{6}{|c|}{ CULTURA ORGANIZACIONAL X ESTRESSE OCUPACIONAL } \\
\hline Ano & Autores & Participantes & $\begin{array}{l}\text { Escala } \\
\text { Cultura }\end{array}$ & $\begin{array}{l}\text { Escala } \\
\text { Estresse }\end{array}$ & Resultados Significativos \\
\hline 2005 & $\begin{array}{l}\text { Tamayo; } \\
\text { Paschoal }^{26}\end{array}$ & 237 bancários & EVRT & EET & $\begin{array}{l}\text { Sem relaçốes significativas entre } \\
\text { cultura e estresse }\end{array}$ \\
\hline 2007 & Tamayo $^{27}$ & 626 bancários & IPVO & EET & $\begin{array}{l}\text { Autonomia } \times \text { Estresse }(\beta=-0,14)_{i} \\
\text { Preocupação com coletividade } x \\
\text { Estresse }(\beta=-0,31) ; \\
\text { Realização } \times \text { Estresse }(\beta=-0,25)_{i} \\
\text { Conformidade } x \text { Estresse }(\beta=0,16) \text {. }\end{array}$ \\
\hline 2010 & $\begin{array}{l}\text { Canova; } \\
\text { Porto }^{12}\end{array}$ & $\begin{array}{l}321 \text { professores } \\
\text { do ensino médio }\end{array}$ & IPVO & EET & $\begin{array}{l}\text { Preocupação com coletividade } x \\
\text { Estresse }(\beta=-0,22) \text {. }\end{array}$ \\
\hline 2010 & Lucena $^{28}$ & $\begin{array}{l}281 \text { professores } \\
\text { do ensino } \\
\text { superior }\end{array}$ & IBACO & ERI & $\begin{array}{l}\text { Sem relações significativas entre } \\
\text { cultura e estresse }\end{array}$ \\
\hline 2014 & $\begin{array}{l}\text { Feitosa; } \\
\text { Belo; } \\
\text { Silva }^{29}\end{array}$ & $\begin{array}{l}104 \text { técnicos de } \\
\text { enfermagem }\end{array}$ & $\begin{array}{l}\text { IBACO } \\
\text { (versão } \\
\text { reduzida) }\end{array}$ & EET & $\begin{array}{l}\text { Sem relações significativas entre } \\
\text { cultura e estresse }\end{array}$ \\
\hline
\end{tabular}

CULTURA ORGANIZACIONAL X BURNOUT

\begin{tabular}{|c|c|c|c|c|c|}
\hline Ano & Autores & Participantes & $\begin{array}{l}\text { Escala } \\
\text { Cultura }\end{array}$ & $\begin{array}{l}\text { Escala } \\
\text { Burnout }\end{array}$ & Resultados Significativos \\
\hline 2002 & $\begin{array}{l}\text { Borges; } \\
\text { Argolo; } \\
\text { Pereira; } \\
\text { Machado; } \\
\text { Silva }{ }^{30}\end{array}$ & $\begin{array}{l}205 \\
\text { profissionais de } \\
\text { saúde }\end{array}$ & EVO & $\mathrm{MBI}$ & $\begin{array}{l}\text { Conservação } \times \text { Burnout }(\beta=-0,28) ; \\
\text { Igualitarismo } \times \text { Exaustão emocional } \\
(\beta=-0,3)_{i} \\
\text { Hierarquia } \times \text { Baixa realização } \\
\text { profissional }(\beta=0,18)_{i} \\
\text { Igualitarismo } \times \text { Baixa realização } \\
\text { profissional }(\beta=-0,32)_{i} \\
\text { Domínio } \times \text { Baixa realização } \\
\text { profissional }(\beta=0,23)_{i} \\
\text { Conservação } \times \text { Despersonalização } \\
(\beta=-0,28) \text {. }\end{array}$ \\
\hline 2011 & Marques $^{24}$ & $\begin{array}{l}45 \text { funcionários } \\
\text { de uma rede } \\
\text { hoteleira }\end{array}$ & QCO & $\mathrm{MBI}$ & $\begin{array}{l}\text { Identificação com a empresa } x \\
\text { Exaustấo emocional }(r=-0,30) \text {; } \\
\text { Benefícios sociais } x \\
\text { Despersonalização }(r=0,41) \text {. }\end{array}$ \\
\hline 2012 & $\begin{array}{l}\text { Corso; }_{i} \\
\text { Santos; } \\
\text { Faller }^{31}\end{array}$ & $\begin{array}{l}35 \text { profissionais } \\
\text { de saúde }\end{array}$ & IVO & $\mathrm{MBI}$ & $\begin{array}{l}\text { Harmonia x Burnout }\left(r_{s}=-0,42\right) ; \\
\text { Domínio x Burnout }\left(r_{s}=-0,42\right) \text {. }\end{array}$ \\
\hline
\end{tabular}

Nota: EVRT= Escala de Valores Relativos ao Trabalho; IPVO = Inventário de Práticas e Valores Organizacionais; IBACO = Instrumento Brasileiro para Avaliação da Cultura Organizacional; EET = Escala de Estresse no Trabalho; ERI = Questionário Esforço e Recompensa no Trabalho; EVO = Escala de valores Organizacionais; $Q C O=$ Questionários de Cultura Organizacional; IVO = Inventário de Valores Organizacionais; MBI = Maslach Burnout Inventory. 
No que se refere aos estudos que envolvem 0 estresse ocupacional, o que se nota é que, embora exista, atualmente, uma diversidade de estudos sobre esse conceito nos mais diversos âmbitos, as suas relações com a cultura organizacional ainda são pouco exploradas no Brasil. Ademais, é válido registrar que, dentre as pesquisas localizadas, muitas limitam-se a apenas um dos elementos que compõem a cultura organizacional: os valores.

Tamayo e Paschoal (2005), por exemplo, em uma pesquisa realizada com 237 funcionários de uma instituição bancária mencionam a inexistência de relações significativas entre valores no trabalho e o estresse ocupacional. Já em estudo posterior, Tamayo (2007), ao testar as relações entre valores organizacionais e estresse em uma amostra de 626 funcionários de algumas empresas bancárias encontrou que os fatores coletividade, realização e autonomia apresentaram relação negativa com 0 estresse enquanto o fator conformidade apresentou correlação positiva com o mesmo fenômeno. Tais achados sugerem que as metas relacionadas a esse construto estão atreladas ao controle e sacrifício contínuo dos funcionários, que periodicamente são exigidas pela conformidade. Assim, partindo do pressuposto que os valores de conformidade regulam os comportamentos e atuam no controle dos desejos pessoais, os resultados indicam que a prioridade da organização em relação a determinados valores pode se manifestar como agente estressor ou mecanismo de prevenção do estresse.

Canova e Porto (2010), por sua vez, dedicaramse à investigação do poder preditivo dos valores organizacionais sobre o nível de estresse ocupacional entre professores do ensino médio. Os resultados apontaram que os valores organizacionais são capazes de predizer o grau de estresse dos trabalhadores através das dimensões ética, preocupação com a coletividade e autonomia, o que sugere que organizações que têm como metas a promoção de justiça, de igualdade no trabalho, de tolerância, de honestidade e são mais voltadas para - coletivismo favorecem que seus trabalhadores não percebam - ou percebam em menor grau - o estresse ocupacional.

Dos estudos apresentados até aqui, fica evidenciada a pouca coerência empírica entre os autores nos mapeamentos realizados sobre as relações estabelecidas entre $o$ estresse ocupacional e os valores organizacionais. Para Tamayo e Pascoal (2005), uma das razões para as incoerências empíricas do campo encontra-se nas diferentes perspectivas adotadas para a conceituação do que são os valores: enquanto alguns autores tratam - tema a partir da perspectiva organizacional (ou seja, valores organizacionais), outros partem da perspectiva individual (valores pessoais no trabalho). Tais diferenças ficam nítidas através do uso de diferentes escalas para a mensuração dos valores: enquanto Tamayo (2007) e Canova e Porto (2010) adotam como instrumento o Inventário de Perfis de Valores Organizacionais (IPVO), Tamayo e Paschoal (2005) utilizam a Escala de Valores Relativos ao Trabalho (EVRT). Trata-se de medidas substancialmente distintas, sendo a IPVO composta por nove fatores - realização, conformidade, domínio, prestígio organizacional, bem-estar dos empregados, tradição, autonomia, preocupação com a coletividade (Oliveira, \& Tamayo, 2004) - e a EVRT composta por apenas quatro dimensões realização no trabalho, relações sociais, prestígio, estabilidade (Porto, \& Tamayo, 2003).

Partindo para os estudos que se propõem a abarcar a cultura organizacional de forma mais abrangente e completa, cabe registrar as recentes pesquisas realizadas com docentes de instituições privadas do ensino superior do município de Guarulhos (Lucena, 2010) e com técnicos em enfermagem de um hospital público do estado do Piauí (Feitosa, Belo, \& Silva, 2014). Ambos os estudos adotaram o Instrumento Brasileiro para a Análise da Cultura Organizacional (IBACO), que se caracteriza abarcar tanto valores (profissionalismo cooperativo, profissionalismo competitivo e a satisfação e bem-estar dos empregados) quanto práticas organizacionais (integração externa, a recompensa e o treinamento e a promoção do relacionamento interpessoal). No primeiro estudo mencionado, os resultados obtidos sugeriram a inexistência de relações significativas entre a cultura organizacional e $\circ$ estresse ocupacional e, no segundo, embora a correlação entre estresse e cultura tenha sido estatisticamente significativa, a magnitude da relação mostrouse bastante baixa, indicando que muito pouco do nível de estresse pode ser esclarecido a partir da variação da cultura organizacional. 
Em relação aos estudos que se propõem a compreender as relações estabelecidas entre a cultura e a síndrome do Burnout, é possível notar, inicialmente, que se trata de campo com escassez de estudos ainda maior do que aquele que cerca as questões relativas à cultura e estresse (Figura 2). A partir de uma perspectiva psicossocial, Borges, Argolo, Pereira, Machado e Silva (2002) investigaram a relação entre os valores atribuídos às organizações por profissionais de saúde e os níveis da síndrome de Burnout em três hospitais universitários do Rio Grande do Norte. Os autores concluíram que os polos axiológicos dos valores organizacionais relacionados à síndrome dependem da configuração da cultura organizacional de cada instituição e de seus conflitos. Dessa forma, organizações que se caracterizem por frequentes conflitos de valores tenderão a ser territórios mais propícios para a emergência da relação entre cultura o burnout.

Já no estudo de Corso, Santos e Faller (2012) também realizado com profissionais de saúde, foi verificada a relação entre os valores organizacionais e a incidência da síndrome de Burnout em equipes de enfermagem de Pronto-Atendimentos Públicos de Saúde no município de Santa Maria/RS. Os resultados apontaram para uma correlação negativa entre os valores domínio e harmonia e a incidência da síndrome de Burnout, sugerindo que tais valores possuem papel importante na ocorrência de problemas de saúde relacionados ao trabalho, assim como constatou o estudo anterior.

Além de estudos com profissionais de saúde foram encontradas pesquisas realizadas com outras categorias profissionais, como, por exemplo, a realizada por Marques (2011) com trabalhadores do ramo hoteleiro de Portugal. Nesta pesquisa, as três dimensões do burnout tiveram as suas associações testadas com diferentes aspectos da cultura organizacional. Em seus resultados, a autora obteve que colaboradores que percebem sua organização como coesa e que se identificam com o papel estratégico da empresa tendem a não apresentar sintomas de exaustão emocional e física, bem como colaboradores que percebem a sua remuneração e benefícios sociais recebidos como justos tendem a não manifestar despersonalização na interação com os clientes.
Assim como aconteceu com o estresse ocupacional, é válido registrar a utilização de diferentes instrumentos de medida para a apreensão da cultura organizacional: 0 estudo de Borges et al. (2002) adotou uma medida proposta por Tamayo e Gondim (1996), a Escala de Valores Organizacionais (EVO); já a pesquisa de Corso et al. (2012) utilizou o Inventário de Valores Organizacionais (IVO); por fim, Marques (2011) optou pela aplicação uma medida de cultura proposta por Sousa (2007). A primeira medida mencionada é composta por seis dimensões que medem os valores organizacionais segundo três polos axiológicos dos valores (autonomia versus conservadorismo, hierarquia versus igualitarismo e domínio versus harmonia). Já o IVO apreende seis aspectos da dinâmica organizacional, sendo eles, agrupados em três eixos axiológicos bipolares: autonomia versus conservadorismo; hierarquia versus estrutura igualitária; e harmonia versus domínio (Tamayo, Mendes, \& Paz, 2000). Por fim, a escala de Sousa (2007) caracteriza-se por ser composta por oito conjuntos de itens, que, em conjunto, analisam diversos aspectos organizacionais: a empresa como um todo, a função, o chefe direto, colegas de setor, a remuneração e benefícios sociais, participação em ações de formação, perspectivas de desenvolvimento e valores organizacionais.

Apesar das diferentes ferramentas de mensuração, os três estudos contemplados na presente revisão obtiveram resultados congruentes: em todos eles, foi possível demostrar a existência de relação entre a síndrome de Burnout, ou pelo menos alguma(s) de suas dimensões, e a cultura organizacional, sendo esta última apreendida a partir dos valores organizacionais ou de outros aspectos presentes na organização, tais como remuneração, benefícios, sistema de gestão e competitividade.

\section{CONSIDERAÇÕES FINAIS}

Em qualquer ambiente organizacional, ter conhecimento a respeito da Cultura Organizacional é de extrema importância, uma vez que seus impactos sobre o estado de adoecimento dos sujeitos no espaço de trabalho manifestam-se como elementos essenciais para o entendimento da 
dinâmica organizacional e, ainda, para a eficiência das políticas e ações a serem implementadas.

Diante do exposto, o presente artigo teve o objetivo de realizar uma revisão da produção nacional entre os anos de 2000 e 2015 que se dedica ao estudo das possíveis relações estabelecidas entre a cultura organizacional - ou alguns de seus aspectos - e o adoecimento psíquico do trabalhador. Esse último conceito foi apreendido por meio de variáveis amplamente presentes nas organizacionais atuais: 0 estresse ocupacional e a síndrome de burnout.

Os resultados evidenciaram a escassez de estudos sobre o tema. Além da escassez, torna-se uma preocupação o fato de poucas pesquisas realizadas se debruçam no estudo da cultura organizacional em sua totalidade dimensional, antes, estão em sua maioria limitadas em um de seus componentes - os valores organizacionais. Essa evidência presente na literatura corrobora para a legitimação das brechas existentes sobre a exploração do tema, levando os pesquisadores a assumirem posturas diversificas, e até mesmo dicotômicas sobre o mesmo fenômeno.

Além disso, um aspecto interessante explicitado neste estudo se refere à dificuldade de articulação dos estudos, o que pode ser atribuído, em alguma medida, à utilização de diferentes ferramentas de mensuração para a apreensão dos mesmos fenômenos. Apesar dos desafios gerados pelas inconsistências empíricas, foi possível identificar que a cultura organizacional configura-se como um preditor do desenvolvimento do estresse ocupacional e da síndrome de burnout, o que corrobora com a postura de, mais do que variáveis disposicionais, é preciso conhecer e gerir variáveis ligadas ao contexto ocupacional - e algumas vezes invisíveis para a promoção da saúde do trabalhador.

Por fim, é importante salientar como limitação do estudo, que as escassas pesquisas teóricas que envolvem a relação do estresse/burnout com a cultura organizacional, fragiliza o processo de coleta de dados, e por conseguinte, $\circ$ aprofundamento das análises realizadas. Assim sendo, como forma de agregar novos horizontes de pesquisa, sugere-se que novos estudos sejam realizados com o intuito de investigar a problemática em questão.

\section{CONTRIBUIÇÕES DOS AUTORES}

Carvalho BR, Ferreira JCM e de Jesus KCO participaram do levantamento de dados, assim como da confecção das análises e da redação do manuscrito. Aguiar CVN e Costa e Silva EE orientaram a condução do trabalho, além de terem contribuído com a redação e revisão do manuscrito final.

\section{CONFLITOS DE INTERESSES}

Nenhum conflito financeiro, legal ou político envolvendo terceiros (governo, empresas e fundações privadas, etc.) foi declarado para nenhum aspecto do trabalho submetido (incluindo mas não limitandose a subvenções e financiamentos, conselho consultivo, desenho de estudo, preparação de manuscrito, análise estatística, etc).

\section{REFERÊNCIAS}

Borges, L.O., Argolo, J.C.T., Pereira, A.L., Machado, E.A.P., \& Silva, W.S. (2002). A síndrome de burnout e os valores organizacionais: um estudo comparativo em hospitais universitários. Psicologia Reflexão e Crítica, 15(1), 189200. doi: $10.1590 /$ S0102-79722002000100020

Canova, K., \& Porto J. (2010). O impacto dos valores organizacionais no estresse ocupacional: um estudo com professores de ensino médio. Revista Administração Mackenzie, 11 (5), 4-31. doi: 10.1590/S167869712010000500002

Codo, W., Soratto, L., \& Vasques-Menezes, I. (2004). Saúde mental e trabalho In: Zanelli, J.C., Borges-Andrade, J., \& Bastos, A.V.B. (Orgs.). Psicologia, organizações e trabalho no Brasil. Porto Alegre: Artmed.

Corso, K.B., Santos, D.L., \& Faller, L.P. (2012). Síndrome de burnout nas organizações públicas de saúde e os valores organizacionais. Revista Administração FACES, 1(1), 88-107. doi: 10.21714/1984-6975FACES2012V11N1ART1 132

Cunha, M.P. (2014). Cultura: O software mental. In: Cunha, M.P., Rego, A., Cunha, R.C., Cabral-Cardoso, C., \& Neves, P. (Orgs.). Manual de comportamento organizacional e gestão. Lisboa: RH Magazine.

Feitosa C., Belo, R., \& Silva C. (2014). A influência da cultura organizacional sobre os níveis de estresse laboral dos técnicos em enfermagem. Revista Perspectivas em Psicologia, 18(1), 75-96.

Gasparini, A.C.L.F., \& Rodrigues, A.L. (1992). Uma perspectiva psicossocial em psicossomática: via estresse e trabalho. In: Mello Filho, J. (Org.). Psicossomática hoje. Porto Alegre: Artes Médicas.

Guimarães, L.A.M., \& Cardoso, W.L.C.D. (2004). Atualizações da síndrome de burnout. São Paulo: Casa do Psicólogo. 
Helman, C.G. (1994). Cultura, saúde e doença. Porto Alegre: Artes Médicas.

Houaiss, A., Villar, M.S., \& Franco, F.M.M. (2001). Dicionário Houaiss da Língua Portuguesa, Rio de Janeiro: Objetiva.

Loureiro, H., Oliveira, A., \& Pessoa, A. (2008). Burnout no trabalho. Revista Referência, 2(7), 33-41.

Lucena, E.S. (2010). Cultura organizacional e estresse ocupacional: um estudo com docentes das instituições privadas de ensino superior do município de Guarulhos. Dissertação de Mestrado em Gestão e Desenvolvimento Organizacional. Departamento de Economia, Contabilidade e Administração. Universidade Taubaté, São Paulo, Brasil.

Mallmann, C.S., Palazzo, L., Carlotto, M.S., \& Castro, D.R.G. (2009). Fatores associados à síndrome de burnout em funcionários públicos municipais. Psicologia: Teoria e Prática, 11 (2), 62-89.

Marques, F.M.P (201 1). As percepções da cultura organizacional e a síndrome de burnout. Dissertação de Mestrado em Gestão de Recursos Humanos. Instituto Superior de Economia e Gestão. Universidade Técnica de Lisboa. Lisboa, Portugal.

Maslach, C. (2007). Entendendo o burnout. In: Rossi, A.M., Perrewe, P.L., \& Sauter, S. (Orgs.). Stress e qualidade de vida no trabalho. São Paulo: Atlas.

Motta, F.P. (1995). Cultura e organizações no Brasil. In: Motta, F.P., \& Caldas, M.P. (Orgs). Cultura organizacional e cultura brasileira. São Paulo: Atlas, 1995.

Mussolin, E., \& Rodas, T. (2013). Avaliação do clima organizacional e estresse no trabalho em uma empresa de grande porte. Revista Científica Eletrônica UNISEB, $1(2)$, 93-105.

Nelson, D. L., \& Simmons, B.L. (2012). Eustresse e esperança no trabalho: mapeando a jornada. In: Rossi, A.M., Perrewé, P.L., \& Sauter, S.L (Orgs.). Stress e qualidade de vida no trabalho: perspectivas atuais da saúde ocupacional. São Paulo: Atlas.

Oliveira, A.F., \& Tamayo, A. (2004). Inventário de perfis de valores organizacionais. Revista de Administração de Empresas, 39(2), 129-140.

Pereira, A.B. (2002). Burnout: quando o trabalho ameaça o bemestar do trabalhador. São Paulo: Casa do Psicólogo.

Porto, J.B., \& Tamayo, A. (2003). Escala de valores relativos ao trabalho - EVT. Psicologia: Teoria e Pesquisa, 19(2), 145152. doi: $10.1590 /$ S0102-37722003000200006

Santos, A.F., \& Cardoso, C.L. (2010). Profissionais de saúde mental: manifestação de stress e burnout. Estudos de Psicologia, 27(1), 67-74. doi: 10.1590/S0103166X2010000100008
Schein, E.H. (2009). Cultura organizacional e liderança. São Paulo: Editora Atlas.

Silva, N., Zanelli, J.C., \& Tolfo, S.R. (2013). Diagnóstico e gestão da cultura nas organizações. In: Borges, L.O., \& Mourão, L. (Orgs.). O trabalho e as organizações: atuações a partir da psicologia. Porto Alegre: Artmed.

Silva, N. (2015). Cultura organizacional e clima organizacional. In: Bendassolli, P.F., \& Borges-Andrade, J.E. (Orgs.). Dicionário da psicologia. São Paulo: Casa do Psicólogo.

Sousa, M.T. (2007). Valor acrescentado da cultura de empresa. Dissertação de Mestrado. Instituto Superior de Economia e Gestão. Universidade Técnica de Lisboa. Lisboa, Portugal.

Spector, P.E. (2002). Psicologia nas organizações. São Paulo: Saraiva.

Tamayo, A. (2007). Impacto dos valores da organização sobre o estresse ocupacional. RAC-Eletrônica, 1(2), 20-33.

Tamayo, A., \& Gondim, S.M.G. (1996). Escala de valores organizacionais. Revista de Administração, 31(2), 62-72.

Tamayo, A., Lima, D., \& Silva, A.V. (2004). Clima organizacional e estresse no trabalho. In: Tamayo, A. (Org.). Cultura e saúde nas organizações. Porto Alegre: Artmed.

Tamayo, A., Mendes, A.M, \& Paz, M.G.T. (2000). Inventário de valores organizacionais. Estudos de Psicologia, 5(2), 289315.

Tamayo, A., \& Paschoal T. (2005). O impacto dos valores laborais e da interferência familiar - trabalho no estresse ocupacional. Psicologia, Teoria e Pesquisa, 21 (2), 173-180. doi: $10.1590 /$ S0102-37722005000200007

Tavares, J.P., Tadeucci, M.S.R., \& Inocente, N.J. (2011). Cultura organizacional e o estresse ocupacional. In: XIV Encontro Latino Americano de Iniciação Científica e X Encontro Latino Americano de Pós-Graduação. Paraíba, UNIVAP.

Tironi, M.O.S. (2005). A síndrome de burnout em médicos pediatras: um estudo em duas organizações hospitalares. Dissertação de Mestrado. Instituto de Psicologia, Universidade Federal da Bahia, Bahia.

Zanelli, J.C., \& Silva, N. (2014). Cultura Organizacional. In: Zanelli, J.C., Borges-Andrade, J.E., \& Bastos, A.V.B. (Orgs.). Psicologia, organizações e trabalho no Brasil. São Paulo: Artmed. 